\title{
Multiple spin-flop phase diagram of $\mathrm{BaCu}_{2} \mathrm{Si}_{2} \mathrm{O}_{7}$.
}

\author{
V.N.Glazkov ${ }^{1,2}$, G.Dhalenne ${ }^{3}$ and A.Revcolevschi ${ }^{3}$, \\ A.Zheludev ${ }^{1}$ \\ ${ }^{1}$ Laboratorium für Festkörperphysik, ETH Zurich, 8093 Zürich, Schweiz \\ 2 P. L. Kapitza Institute for Physical Problems RAS, 119334 Moscow, Russia \\ ${ }^{3}$ Laboratoire de Physico-Chimie de l'Etat Solide, Université Paris-Sud, 91405 Orsay \\ cedex, France \\ E-mail: glazkov@kapitza.ras.ru
}

\begin{abstract}
The quasi one-dimensional compound $\mathrm{BaCu}_{2} \mathrm{Si}_{2} \mathrm{O}_{7}$ demonstrates numerous spin-reorientation transitions both for a magnetic field applied along the easy axis of magnetization, and for a magnetic field applied perpendicular to it. The magnetic phase diagram for all three principal orientations is obtained by magnetization and specific heat measurements. Values of all critical fields and low-temperature values of magnetization jumps are determined for all transitions.

PACS numbers: 75.30.Kz, 75.50.Ee
\end{abstract}

Submitted to: J. Phys.: Condens. Matter

\section{Introduction.}

A spin-flop transition is a well-known characteristic feature of easy-axis antiferromagnets. It is caused by a competition between the anisotropy energy, which is minimized by aligning the order parameter along the easy axis, and the Zeeman energy, which is minimized for an antiferromagnet by aligning the order parameter perpendicularly to the field. Thus, at a certain field value, the order parameter re-orients itself, going away from the easy axis. For a collinear antiferromagnet only one transition of such sort is usually observed when the magnetic field is applied along the easy axis.

The quasi-one-dimensional compound $\mathrm{BaCu}_{2} \mathrm{Si}_{2} \mathrm{O}_{7}$ has attracted much interest due to its "excessive" spin-flop transitions. The main exchange integral in this compound is $\mathrm{J}=24.1 \mathrm{meV}$, while inter-chain interactions are a factor of 100 smaller [1]. It orders antiferromagnetically at $T_{N}=9.2 \mathrm{~K}$. The ordered local magnetic moment at zero field was found to be equal to $0.15 \mu_{B}[1$. The easy axis of magnetization is aligned along the $c$ direction of the orthorhombic crystal. This is confirmed by magnetization measurements [2] and by neutron diffraction results [3]. Instead of the expected single spin-flop transition, two spin-flop transitions were observed on the magnetization curves when the field was applied along the easy axis $\mathbf{H} \| c[2]$ at $\mu_{0} H_{c 1}=2.0 \mathrm{~T}$ and $\mu_{0} H_{c 2}=4.9 \mathrm{~T}$. 


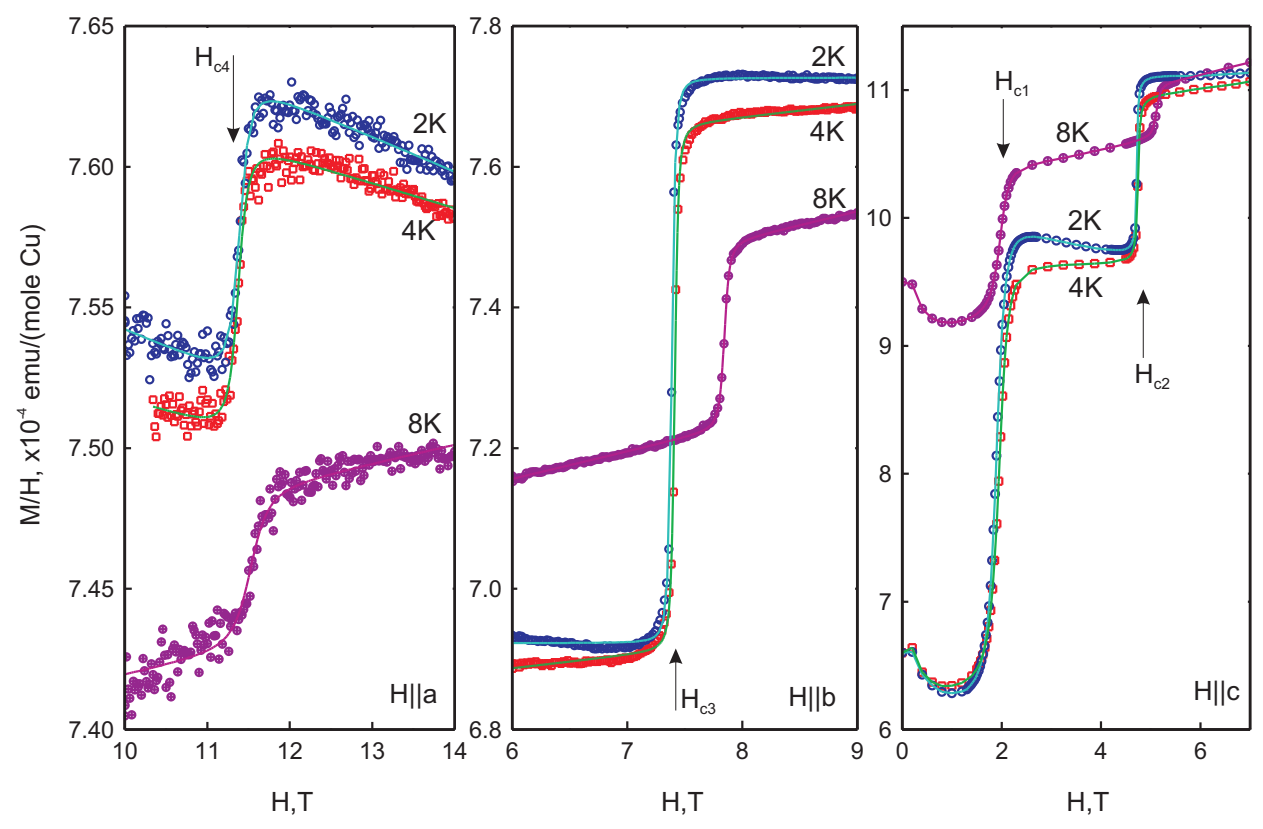

Figure 1. Field dependences of the $\mathrm{M} / \mathrm{H}$ ratio in different orientations. The curves are guides for the eye.

Later, ultrasonic studies [4] have revealed another phase transition when the field was applied perpendicularly to the easy axis $\mathbf{H} \| b$, with a critical field value of $\mu_{0} H_{c 3}=7.8 \mathrm{~T}$. Finally, a spin-reorientation transition in the third orientation $\mathbf{H} \| a$ was observed by electron-spin-resonance [5] with a critical field of $\mu_{0} H_{c 4}=11 \mathrm{~T}$. The exact reason for these numerous phase transitions is not completely clear yet. It was suggested that a strong reduction of the ordered moment could increase the importance of the anisotropy of the transverse susceptibility [5].

Despite these unusual properties, a detailed characterization of the magnetic phase diagram of this compound remains incomplete. The phase diagram in the $\mathbf{H} \| c$ orientation was determined by magnetization measurements [2] and ultrasonic studies [4]. For the $\mathbf{H} \| b$ orientation, only ultrasonic data are available [4]. Finally, the phase transition in the $\mathbf{H} \| a$ orientation was reported only at a temperature of $1.5 \mathrm{~K}$ in electronspin resonance experiments [5]. No magnetization studies of the phase transitions for $\mathbf{H} \| a, b$ were reported to date.

In the present paper we fill in these gaps and report results of magnetization and specific heat studies of the phase diagram of $\mathrm{BaCu}_{2} \mathrm{Si}_{2} \mathrm{O}_{7}$.

\section{Sample preparation and experimental details.}

We used single crystalline samples of $\mathrm{BaCu}_{2} \mathrm{Si}_{2} \mathrm{O}_{7}$ taken from the same batch as those used in Ref.[5]. All measurements were performed on the same sample of approximate size $2 \times 3 \times 0.5 \mathrm{~mm}^{3}$. Sample orientation was checked by X-ray diffraction on a Bruker APEX-II diffractometer. The misalignment of the mounted sample is estimated to be within $5^{\circ}$. 


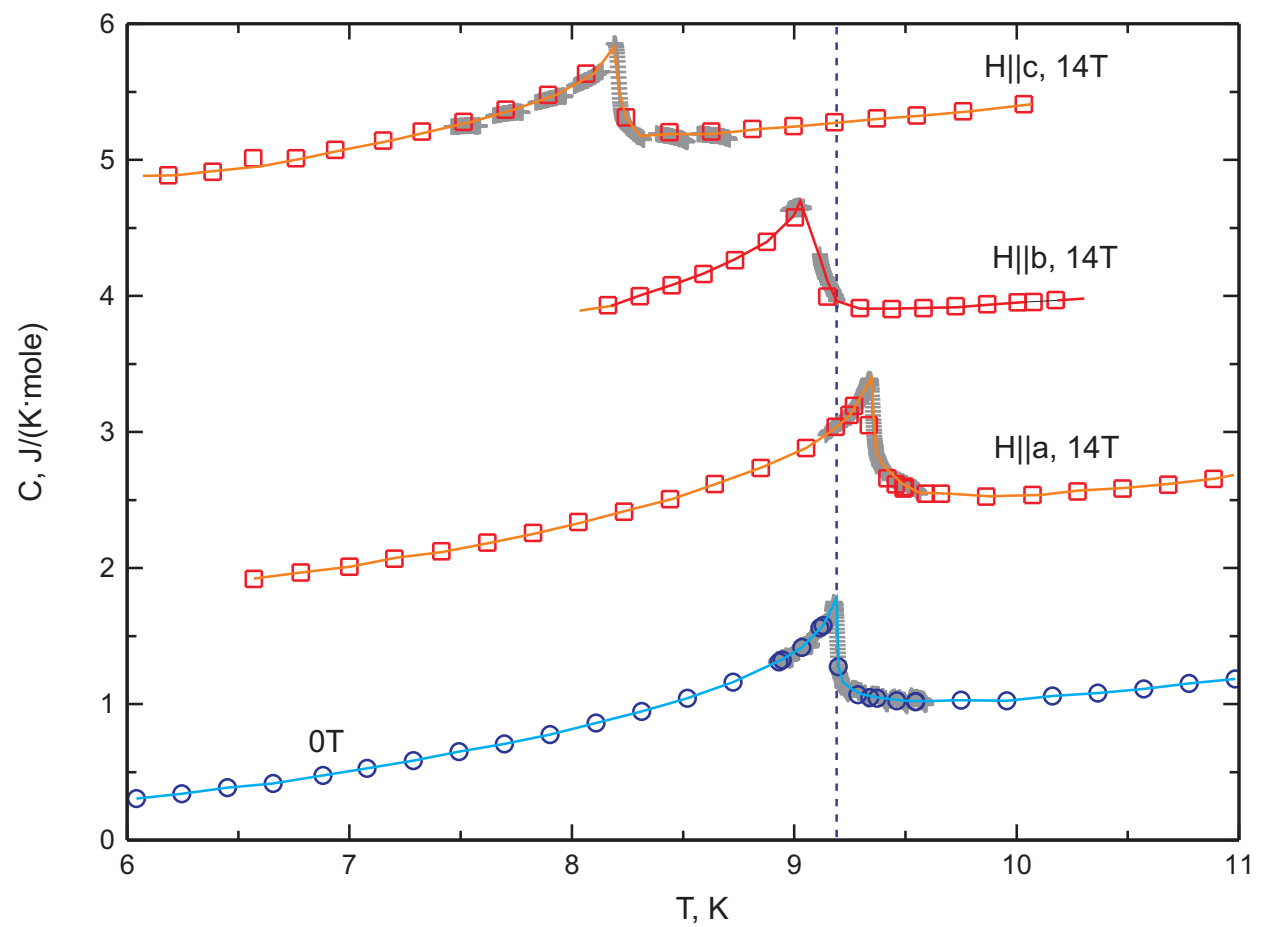

Figure 2. Change of the Néel temperature with field in different orientations. The specific heat curves measured at $14 \mathrm{~T}$ for $\mathbf{H} \| a, b$ and $\mathbf{H} \| c$ are shifted upwards by 1.5, 3.0 and $4.5 \mathrm{~J} /(\mathrm{K} \cdot \mathrm{mole})$, respectively. Crosses: specific heat values obtained from slope analysis of thermal relaxation curves. Circles and squares: specific heat values obtained from best fits in the two- $\tau$ model. Vertical dashed line: position of the phase transition in zero field, $T_{N}=9.19 \mathrm{~K}$. Solid lines: guides for the eye.
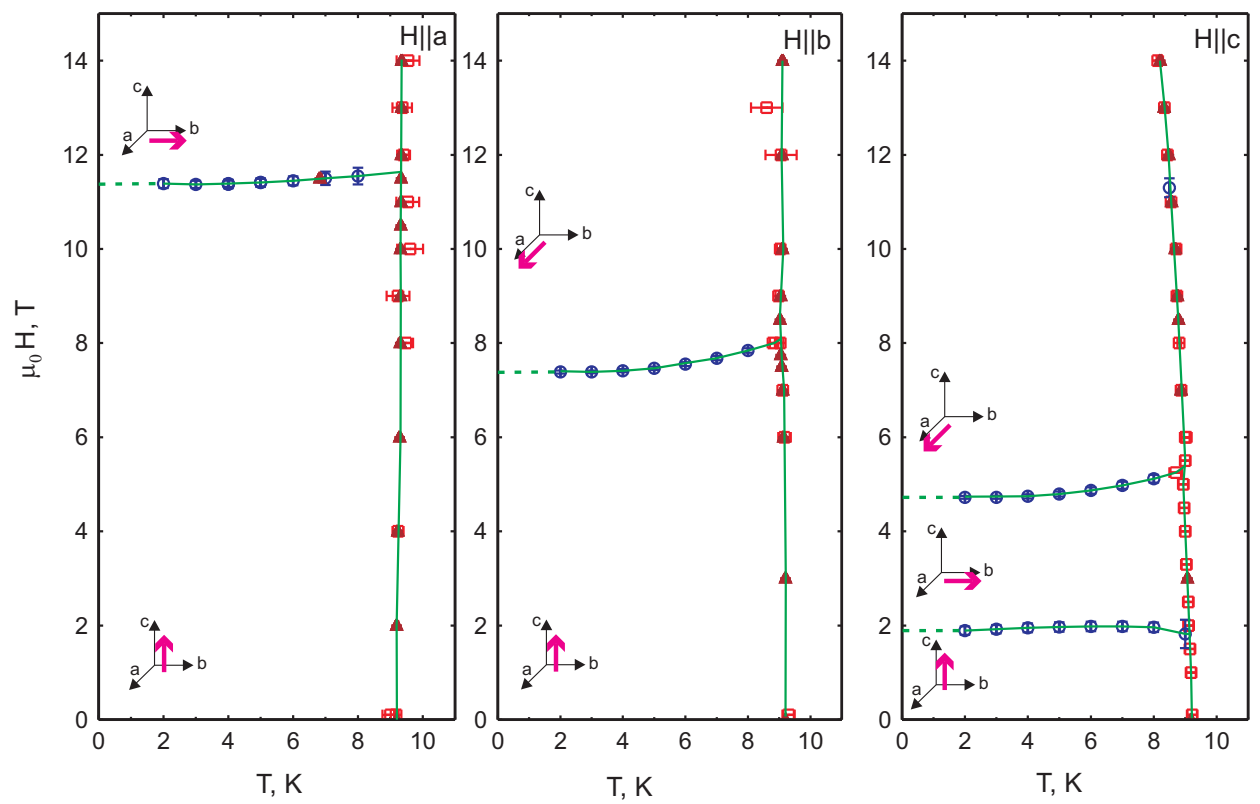

Figure 3. Complete set of phase diagrams for $\mathrm{BaCu}_{2} \mathrm{Si}_{2} \mathrm{O}_{7}$. Circles: data from magnetization field scans. Squares: data from magnetization temperature scans. Triangles: data from specific heat measurements. Solid and dashed lines marking phase boundaries: guides for the eye. Orientation of the antiferromagnetic order parameter in all phases is shown according to Ref. [5] 
Multiple spin-flop phase diagram of $\mathrm{BaCu}_{2} \mathrm{Si}_{2} \mathrm{O}_{7}$.

Magnetization was measured by a Quantum Design MPMS-XL SQUIDmagnetometer and a Quantum Design PPMS system equipped with a vibrating sample magnetometer.

Specific heat was measured using Quantum Design PPMS system. It was measured by applying a controlled heat pulse to the platform with the sample, which is connected to the cryostat with a stable heat-link. The sample temperature relaxation curve $T(t)$ was then fitted by a two- $\tau$ model, yielding the value of the specific heat. To improve the precision of the transition temperature determination, a slope analysis technique was additionally used. This technique makes use of the thermal balance equation $P=C \frac{d T_{\text {sample }}}{d t}+K\left(T_{\text {sample }}-T_{\text {cryo }}\right)$ (where $C$ is the total specific heat of the sample and the platform, $P$ - the heater power, $K$ - the heat link thermal conductivity, $T_{\text {sample }}$ and $T_{\text {cryo }}$ - being the temperatures of the sample and of the cryostat, respectively).

\section{Experimental results and discussion.}

Examples of magnetization and specific heat curves are shown in Figures 1 and 2. Both demonstrate clear anomalies at the phase transitions. This allows to build phase diagrams in all orientations of the applied magnetic field, as shown in Figure 3 ,

The zero-field transition temperature is $\left.T_{N}\right|_{H=0 T}=9.19 \pm 0.01 \mathrm{~K}$. The transition temperature is field dependent, without strong anomalies at the crossings of the phase transition lines. For $\mathbf{H} \| a$, the transition temperature slightly increases with field, while for the other two principal orientations, it decreases with field. The transition temperatures at a field of $14 \mathrm{~T}$ are: for $\left.\mathbf{H}|| a T_{N}\right|_{H=14 T}=9.35 \pm 0.01 \mathrm{~K}$, for $\mathbf{H} \| b$ $\left.T_{N}\right|_{H=14 T}=9.09 \pm 0.02 \mathrm{~K}$, for $\left.\mathbf{H}|| c T_{N}\right|_{H=14 T}=8.21 \pm 0.02 \mathrm{~K}$.

The low-temperature values of the critical fields, as determined by our magnetization measurements, are: for $\mathbf{H} \| c H_{c 1}=(1.89 \pm 0.10) \mathrm{T}, H_{c 2}=(4.72 \pm 0.03) \mathrm{T}$; for $\mathbf{H} \| b H_{c 3}=(7.39 \pm 0.03) \mathrm{T}$; and for $\mathbf{H} \| a H_{c 4}=(11.40 \pm 0.08) \mathrm{T}$. These values are in good agreement with published data [2, 4, 5]. The critical field values are weakly temperature dependent: the corresponding phase transition lines are almost horizontal.

The measured magnetic susceptibilities of the higher-field phases are always higher than those of lower-field phases (see Figure 1). This confirms the identification of the field-induced phase transitions as spin-reorientation transitions, caused by the competition between the order parameter anisotropy and the Zeeman energy. Susceptibility jumps $\Delta \chi=\Delta(M / H)$ at these transitions, as measured at $2 \mathrm{~K}$, are: for $\mathbf{H} \| c(3.54 \pm 0.05) \cdot 10^{-4} \mathrm{emu} /($ mole $\mathrm{Cu})$ and $(1.35 \pm 0.05) \cdot 10^{-4} \mathrm{emu} /($ mole $\mathrm{Cu})$, for the first and second spin-flops, respectively; for $\mathbf{H} \| b(0.813 \pm 0.020) \cdot 10^{-4} \mathrm{emu} /(\mathrm{mole} \mathrm{Cu})$; and for $\mathbf{H} \| a(0.085 \pm 0.020) \cdot 10^{-4} \mathrm{emu} /($ mole $\mathrm{Cu})$.

A model that describes all low-temperature phase transitions and antiferromagnetic resonance frequency-field dependences was proposed in Ref.[5]. This model suggests that anisotropic contributions to the transverse susceptibility are unusually large in $\mathrm{BaCu}_{2} \mathrm{Si}_{2} \mathrm{O}_{7}$, probably due to the strong reduction of the ordered magnetic moment. The low-energy dynamics of the antiferromagnetic order parameter is then described by 
the potential energy:

$$
\begin{aligned}
U= & -\frac{1}{2}[\mathbf{l} \times \mathbf{H}]^{2}+a_{1} l_{x}^{2}+a_{2} l_{y}^{2}+\xi_{1}(\mathbf{H} \cdot \mathbf{l}) H_{x} l_{x}+ \\
& +\xi_{2}(\mathbf{H} \cdot \mathbf{l}) H_{y} l_{y}-\left(\xi_{1}+\xi_{2}\right)(\mathbf{H} \cdot \mathbf{l}) H_{z} l_{z}- \\
& -B_{1} H_{x}^{2}\left(l_{y}^{2}-l_{z}^{2}\right)-B_{2} H_{y}^{2}\left(l_{x}^{2}-l_{z}^{2}\right)-B_{3} H_{z}^{2}\left(l_{x}^{2}-l_{y}^{2}\right)+ \\
& +C_{1} H_{y} H_{z} l_{y} l_{z}+C_{2} H_{x} H_{z} l_{x} l_{z}+C_{3} H_{x} H_{y} l_{x} l_{y}
\end{aligned}
$$

Here, Cartesian coordinates are chosen as $x\|a, y\| b$ and $z \| c$ and $\mathbf{l}$ is the antiferromagnetic order parameter. The exchange part of the transverse susceptibility is set to unity for the sake of convenience. The phase transitions are described in terms of this potential energy as rotations of the order parameter $\mathbf{l}$. In the low-field phases, the order parameter is aligned along the easy axis, $\mathbf{l} \| z$. When the field is applied along the easy axis $z$, it rotates at $H_{c 1}$ towards the second easy axis, $\mathbf{l} \| y$, and at $H_{c 2}$, towards the hard axis $\mathbf{l} \| x$. When the field is applied along $y$ or $x$, the order parameter rotates towards the $x$ or the $y$ axis at $H_{c 3}$ and $H_{c 4}$, respectively. These orientations of the order parameter are shown schematically in Figure 3.

The parameter values, all determined from best fits of the electron spin resonance data [5], are: $\gamma=2.82 \mathrm{GHz} / \mathrm{kOe}, a_{1}=400 \mathrm{kOe}^{2}, a_{2}=118 \mathrm{kOe}^{2}, B_{1}=0.0047, B_{2}=0.0370$, $B_{3}=0.0614, \xi_{1}=0.135, \xi_{2}=-0.03$. The $C_{i}$ constants could be ignored in the principal orientations of the magnetic field.

Our magnetization measurements allow an independent check of this model, since susceptibility jumps at the phase transitions are related to certain parameters of the potential (11). Predicted susceptibility jumps at spin-reorientation transitions are:

$$
\begin{aligned}
& \Delta \chi_{1}=1-2 B_{3}-2\left(\xi_{1}+\xi_{2}\right) \\
& \Delta \chi_{2}=4 B_{3} \\
& \Delta \chi_{3}=4 B_{2} \\
& \Delta \chi_{4}=4 B_{1}
\end{aligned}
$$

Here, $\Delta \chi_{i}$ are susceptibility jumps at the corresponding critical field $H_{c} i$. In order to exclude the scaling factor involved in the choice of energy units in Eqn 1 they can be normalized to $\Delta \chi_{1}$.

A comparison of the calculated and measured values is given below:

\begin{tabular}{|c|c|c|}
\hline & measured & calculated \\
\hline$\Delta \chi_{2} / \Delta \chi_{1}$ & $0.381 \pm 0.015$ & 0.365 \\
$\Delta \chi_{3} / \Delta \chi_{1}$ & $0.230 \pm 0.007$ & 0.220 \\
$\Delta \chi_{4} / \Delta \chi_{1}$ & $0.024 \pm 0.006$ & 0.028 \\
\hline
\end{tabular}

The correspondence between experimental and model values is close to perfect. Thus, the magnetization measurements are fully compatible with the proposed form of potential energy. This supports the model of phase transitions proposed in Ref.[5] and points to the non-trivial effects of spin-reduction on spin-reorientation transitions in low-dimensional antiferromagnets. 


\section{Acknowledgments}

The work was supported by RFBR Grant No.09-02-00736.

[1] M. Kenzelmann, A. Zheludev, S. Raymond, E. Ressouche, T. Masuda, P. Böni, K. Kakurai, I. Tsukada, K. Uchinokura, and R. Coldea, Phys. Rev. B 64, 054422 (2001).

[2] I. Tsukada, J. Takeya, T. Masuda, and K. Uchinokura, Phys. Rev. Lett. 87, 127203 (2001)

[3] A. Zheludev, E. Ressouche, I. Tsukada, T. Masuda, and K. Uchinokura, Phys. Rev. B 65, 174416 (2002)

[4] M. Poirier, M. Castonguay, A. Revcolevschi, and G. Dhalenne, Phys. Rev. B 66, 054402 (2002)

[5] V. N. Glazkov, A. I. Smirnov, A. Revcolevschi, and G. Dhalenne, Phys. Rev. B 72, 104401 (2005) 